\title{
GENERATING SOLUTIONS OF A LINEAR EQUATION AND STRUCTURE OF ELEMENTS OF THE ZELISKO GROUP
}

\author{
V.A. BOVDI AND V.P. SHCHEDRYK
}

\begin{abstract}
Solutions of a linear equation $b=a x$ in a homomorphic image of a commutative Bézout domain of stable range 1.5 is developed. It is proved that the set of solutions of a solvable linear equation contains at least one solution that divides the rest, which is called a generating solution. Generating solutions are pairwise associates. Using this result, the structure of elements of the Zelisko group is investigated.
\end{abstract}

\section{IntRoduCtion AND MAIN RESUlts}

Let $R$ be a commutative elementary divisor ring with $1 \neq 0$ (see [6, p. 465]) and let $R^{n \times n}$ be the ring of $n \times n$ matrices over $R$ in which $n \geq 2$. Let $U(R)$ and $\mathrm{GL}_{n}(R)$ be groups of units of rings $R$ and $R^{n \times n}$, respectively. By the definition [6, p. 465] of elementary divisor rings, for each $A \in R^{n \times n}$, there exist $P, Q \in \mathrm{GL}_{n}(R)$ (we call them left and right transforming matrices of the matrix $A$ ) such that

$$
P A Q=\operatorname{diag}\left(\varphi_{1}, \ldots, \varphi_{k}, 0, \ldots, 0\right),
$$

where $\varphi_{k} \neq 0$ and $\varphi_{i}$ is a divisor of $\varphi_{i+1}$ for $i=1, \ldots, k-1<n$.

The diagonal matrix $\Phi:=\operatorname{diag}\left(\varphi_{1}, \ldots, \varphi_{k}, 0, \ldots, 0\right)$ in (1) is called the Smith form and $\varphi_{1}, \ldots, \varphi_{k}$ are called invariant factors of the matrix $A$. Since invariant factors in (11) are determined uniquely up to associates, the Smith form of $A$ is defined ambiguously.

To the matrix $\Phi$ we associate a subgroup $\mathbf{G}_{\Phi} \leq \mathrm{GL}_{n}(R)$ (see [10, p. 62]) which is called the Zelisko group of the matrix $\Phi$ and it is definite as:

$$
\mathbf{G}_{\Phi}=\left\{H \in \mathrm{GL}_{n}(R) \mid \exists S \in \mathrm{GL}_{n}(R) \text { such that } H \Phi=\Phi S\right\} .
$$

This definition was first given by V. Zelisko [16] for the matrix over polynomial ring $F[x]$ in which $F$ is an algebraic closed field of characteristic 0 . The definition of the Zelisko group $\mathbf{G}_{\Phi}$ over the ring $R$ is independent of the choice of the Smith form $\Phi$ of $A$ (see (10)). Indeed, let $\Phi_{1}:=\Phi \Upsilon$ in which $\Upsilon:=\operatorname{diag}\left(\varepsilon_{1}, \ldots, \varepsilon_{k}, 1, \ldots, 1\right)$ and $\varepsilon_{i} \in U(R)$. If $H \in \mathbf{G}_{\Phi}$ then

$$
H \Phi_{1}=H(\Phi \Upsilon)=\Phi(S \Upsilon)=\Phi \Upsilon\left(\Upsilon^{-1} S \Upsilon\right)=\Phi_{1} S_{1}, \quad\left(S_{1}:=\Upsilon^{-1} S \Upsilon\right)
$$

and $\mathbf{G}_{\Phi} \subseteq \mathbf{G}_{\Phi_{1}}$.

Now, if $L \in \mathbf{G}_{\Phi_{1}}$, then $L \Phi_{1}=\Phi_{1} T$, where $T \in \mathrm{GL}_{n}(R)$, so $L \Phi=\Phi\left(\Upsilon T \Upsilon^{-1}\right)$ and $\mathbf{G}_{\Phi_{1}} \subseteq \mathbf{G}_{\Phi}$. Consequently, $\mathbf{G}_{\Phi_{1}}=\mathbf{G}_{\Phi}$.

Note that, if $R$ is an elementary divisor domain and $\Phi:=\operatorname{diag}\left(\varphi_{1}, \ldots, \varphi_{n}\right)$ in (11) is a nonsingular matrix (i.e. $\operatorname{det}(\Phi) \neq 0$ ), then the group $\mathbf{G}_{\Phi}$ consists (see [10, Theorem 2.6, p. 63]) of

2020 Mathematics Subject Classification. 15A06, 15A21, 13 A05.

Key words and phrases. Linear equation, Commutative Bézout domain, Stable range, Zelisko group. 
all invertible matrices of the following form:

$$
\left[\begin{array}{ccccc}
h_{11} & h_{12} & \ldots & h_{1 . n-1} & h_{1 n} \\
\frac{\varphi_{2}}{\varphi_{1}} h_{21} & h_{22} & \ldots & h_{2 . n-1} & h_{2 n} \\
\ldots & \ldots & \ldots & \ldots & \ldots \\
\frac{\varphi_{n}}{\varphi_{1}} h_{n 1} & \frac{\varphi_{n}}{\varphi_{2}} h_{n 2} & \ldots & \frac{\varphi_{n}}{\varphi_{n-1}} h_{n . n-1} & h_{n n}
\end{array}\right] \in R^{n \times n} .
$$

If for the matrix $A$ we fix $\Phi$ in (1), then the matrices $P$ and $Q$ are also defined ambiguously. As it was shown in [10, Property 2.2, p. 63], the set of left transforming matrices of $A$ coincides with the right coset $\mathbf{G}_{\Phi} P$ of the Zelisko group $\mathbf{G}_{\Phi}$ in $\mathrm{GL}_{n}(R)$. A similar property holds for the set of right transforming matrices of $A$. Moreover, the group $\mathbf{G}_{\Phi}$ actively used in the following.

Fact 1. [10, Theorem 4.3, p. 138] Let $R$ be a commutative elementary divisor domain. Let $P_{A}$ and $P_{B}$ be left transforming matrices of $A, B \in R^{n \times n}$, respectively. If $A$ and $B$ have the same Smith's form $\Phi$, then the following conditions are equivalent:

(i) $A$ and $B$ are right associates, i.e. $A=B U$ for some $U \in \mathrm{GL}_{n}(R)$;

(ii) $P_{B}=H P_{A}$ for some $H \in \mathbf{G}_{\Phi}$;

(iii) $\mathrm{G}_{\Phi} P_{A}=\mathrm{G}_{\Phi} P_{B}$.

We would like to note that the concept of the Zelisko group as well as its properties, were used by Kazimirskiı [7] for the solution of the problem of extraction of a regular divisor of a matrix over the polynomial ring $F[x]$, where $F$ is an algebraically closed field of characteristic 0 . The properties of the group $\mathbf{G}_{\Phi}$ in which $\Phi \in R^{n \times n}$, were explicitly investigated in [10. Chapter 2.2 and Chapter 2.6].

The notation $a \mid b$ in $R$ means that $b=a c$ for some $c \in R$. The greatest common divisor of $a, b \in R$ is denoted by $(a, b)$. The ring $R$ has stable range 1.5 (see [12, p. 961] and [11, p. 46]) if for each $a, b \in R$ and $c \in R \backslash\{0\}$ with the property $(a, b, c)=1$ there exists $r \in R$ such that

$$
(a+b r, c)=1 \text {. }
$$

This notion arose as a modification of the Bass's concept of the stable range of rings (see [2, p. 498]). The examples of rings of stable range 1.5 are Euclidean rings, principal ideal rings, rings of algebraic integers, rings of integer analytic functions, adequate rings [10, p. 20] and [3]. Note that the commutative rings of stable range 1.5 coincide with rings of almost stable range 1 [1, 8].

If the ring $R$ has stable range 1.5, then some properties of the Zelisko group $\mathbf{G}_{\Phi}$ are closely related to a factorizability of the general linear group over $R$ (see [13, Theorem 3, p. 144] and [10, Chapter 2.6]).

The subgroups of the lower- and upper- unitriangular $n \times n$-matrices of the general linear group $\mathrm{GL}_{n}(R)$ are denote by $U_{n}^{l w}(R)$ and $U_{n}^{u p}(R)$, respectively.

Fact 2. 13] If $R$ is a commutative Bézout domain, then the following conditions are equivalent:

(i) $R$ has stable range 1.5 ;

(ii) $\mathrm{GL}_{2}(R)=\mathbf{G}_{\Phi} U_{2}^{l w}(R) U_{2}^{u p}(R)$ for all $\Phi \in \mathrm{GL}_{2}(R)$;

(iii) $\mathrm{GL}_{n}(R)=\mathrm{G}_{\Phi} U_{n}^{l w}(R) U_{n}^{u p}(R)$ for all $\Phi \in \mathrm{GL}_{n}(R)$ in which $n \geq 2$.

Note that Vaserstein and Wheland [15] proved that if $R$ has stable range 1, then

$$
\mathrm{GL}_{n}(R)=G T_{n}^{l w}(R) U_{n}^{u p}(R) U_{n}^{l w}(R), \quad(n \geq 2)
$$

in which $G T_{n}^{l w}(R)$ is the group of invertible lower triangular matrices. In the case of second order matrices over the commutative ring $R$ the converse of this statement was proved in [9]. 
Moreover, it was shown in [9] that a commutative ring $R$ is a Hermite ring of stable range 1 if and only if

$$
M_{n}(R)=T_{n}^{l w}(R) U_{n}^{u p}(R) U_{n}^{l w}(R), \quad(n \geq 2)
$$

where $T_{n}^{l w}(R)$ is the ring of lower triangular $n \times n$ matrices. For the case of a noncommutative ring $R$, this statement was proved in [5, Theorem 1.2.2., p. 12] (see also [4]).

The notion of rings of stable range 1.5 also closely related with complementability of an unimodular row to an invertible matrix.

Fact 3. [13] Let $R$ be a commutative Bézout domain. The following conditions are equivalent:

(i) $R$ has stable range 1.5 ;

(ii) for each relatively prime elements $a_{1}, \ldots, a_{n} \in R$, in which $n \geq 3$ and $a_{1} \neq 0$, there exists an invertible matrix of the following form:

$$
\left[\begin{array}{cccccc}
u_{n} & 0 & \ldots & 0 & 0 & u_{1} \\
0 & 1 & & 0 & 0 & u_{2} \\
\vdots & & \ddots & & \vdots & \vdots \\
0 & 0 & & 1 & 0 & u_{n-2} \\
0 & 0 & \ldots & 0 & 1 & u_{n-1} \\
a_{1} & a_{2} & \ldots & a_{n-2} & a_{n-1} & a_{n}
\end{array}\right] \in R^{n \times n} .
$$

We begin our article by investigating the properties of solutions of linear equations in homomorphic images of a commutative Bézout domain $R$ of stable range 1.5. Those solutions of a solvable linear equation $b=a \cdot x \quad(a, b \in R)$ which divide all other are called generating solutions of this equation.

Our first result is related to generating solutions of linear equations.

Theorem 1. Let $R$ be a commutative Bézout domain (with the property $1 \neq 0$ ) of stable range 1.5. Let $U(R)$ be the group of units of $R$. For each $m \in R \backslash\{U(R), 0\}$ we denote the factor ring $R_{m}=R / m R$. Let $a, b \in R_{m}$. The following conditions hold:

(i) each solvable linear equation $b=a x$ in $R_{m}$ has at least one generating solution;

(ii) each two generating solutions of a solvable linear equation $b=$ ax are pairwise associates.

If we fix an ordering relation $\leq$ on elements of the set $R_{m}$, then the set of generating solutions of each solvable equation $\varphi_{2}=\varphi_{1} \cdot x$ contains a minimal element which we denote by $\frac{\varphi_{2}}{\varphi_{1}}$.

Now we are able to formulate our next result.

Theorem 2. Let $R$ be a commutative Bézout domain (with the property $1 \neq 0$ ) of stable range 1.5. Let $U(R)$ be the group of units of $R$. For each $m \in R \backslash\{U(R), 0\}$ we denote the factor ring $R_{m}=R / m R$. Let $\Phi:=\operatorname{diag}\left(\varphi_{1}, \varphi_{2}, \ldots, \varphi_{n}\right) \in R_{m}^{n \times n}$ in which $\varphi_{1}\left|\varphi_{2}\right| \cdots \mid \varphi_{n} \neq 0$ and $n \geq 2$. The Zelisko group $\mathbf{G}_{\Phi}$ consists of all invertible matrices of the form:

$$
\left[\begin{array}{ccccc}
h_{11} & h_{12} & \cdots & h_{1, n-1} & h_{1 n} \\
\frac{\varphi_{2}}{\varphi_{1}} h_{21} & h_{22} & \cdots & h_{2, n-1} & h_{2 n} \\
\cdots & \cdots & \cdots & \cdots & \cdots \\
\frac{\varphi_{n}}{\varphi_{1}} h_{n 1} & \frac{\varphi_{n}}{\varphi_{2}} h_{n 2} & \cdots & \frac{\varphi_{n}}{\varphi_{n-1}} h_{n, n-1} & h_{n n}
\end{array}\right]
$$

in which $h_{i j} \in R_{m}$ and the element $\frac{\varphi_{k}}{\varphi_{l}} \in R_{m}$ is the minimal generating solution of the linear equation $\varphi_{k}=\varphi_{l} \cdot x$ in $R_{m}$ with $1 \leq l<k \leq n$. 


\section{Preliminaries And Proofs}

Let $U(R)$ be the group of units of a commutative Bézout domain $R$ of stable range 1.5. For each $m \in R \backslash\{0, U(R)\}$ we define the homomorphism $\boldsymbol{\boldsymbol { \sigma }}: R \rightarrow R_{m}=R / m R$. For each $a \in R$, we denote $\bar{a}:=\bar{\bullet}(a) \in R_{m}$.

We start our proof with the following.

Lemma 1. Let $\alpha, \beta, \sigma \in R$ such that $\bar{\alpha}=\bar{\beta} \cdot \bar{\sigma}$. There exist $a, b, c \in R$, such that

$$
a=b \cdot c, \quad \bar{a}=\bar{\alpha}, \quad \bar{b}=\bar{\beta}, \quad \text { and } \quad \bar{c}=\bar{\sigma} .
$$

Proof. Set $\bar{a}:=\alpha+m R, \bar{b}:=\beta+m R$ and $\bar{c}:=\sigma+m R$. Since $\beta \sigma \in \alpha+m R$, there exists $t \in R$ such that $\beta \sigma=\alpha+m t$. Put $a:=\alpha+m t, b:=\beta$ and $c:=\sigma$.

Lemma 2. Let $a, b \in R$. Elements $\bar{a}$ and $\bar{b}$ are associates in $R_{m}$ if and only if $(a, m)=(b, m)$.

Proof. If $\bar{a}=\bar{b} \cdot \bar{c}$, then there are always exist $a, b, c \in R$ such that $a=b c$ by Lemma 1. We will use this fact freely. Set $\mu_{a}:=(a, m)$ and $\mu_{b}:=(b, m)$.

$\Leftarrow$. Clearly, $a=\mu_{a} a_{1}$ and $m=\mu_{a} m_{1}$, where $\left(a_{1}, m_{1}\right)=1$ and $a_{1}, m_{1} \in R$. Thus, there exist $u, v \in R$ such that $a_{1} u+m_{1} v=1$ and for any $r \in R$ we have

$$
a_{1}\left(u+r m_{1}\right)+m_{1}\left(v-r a_{1}\right)=1 .
$$

As $\left(u, m_{1}\right)=1$, this means that $\left(u, m_{1}, m\right)=1$. Since $R$ is a commutative Bézout domain of stable range 1.5 and $m \neq 0$, there exists $r_{0} \in R$ such that $\left(u+r_{0} m_{1}, m\right)=1$. Hence $\overline{u+r_{0} m_{1}} \in U\left(R_{m}\right)$. Putting $r=r_{0}$ in (3) and multiplying by $\mu_{a}$ we get that

$$
a\left(u+r_{0} m_{1}\right)+m\left(v-r_{0} a_{1}\right)=\mu_{a},
$$

so $\bar{a}$ and $\bar{\mu}_{a}$ are associates in $R_{m}$. Repeating the same reasoning, we get $\bar{b}$ and $\bar{\mu}_{a}$ are associates in $R_{m}$. By transitivity of the associability relation, the elements $\bar{a}$ and $\bar{b}$ are also associates.

$\Rightarrow$. Let $\bar{a}=\bar{b} \cdot \bar{e}$, where $\bar{e} \in U\left(R_{m}\right)$. There exist $a, b \in R$, such that $a=b e$ in which $(e, m)=1$ by Lemma 1. Consequently, $(a, m)=(b e, m)=(b, m)$.

Lemma 3. Any element $\bar{a}$ in $R_{m}$ can be written as $\bar{a}=\bar{\mu}_{a} \bar{e}_{a}$, where $\mu_{a}:=(a, m)$ is a preimage of $\bar{\mu}_{a}$, a is a preimage of $\bar{a}$, and $\bar{e}_{a} \in U\left(R_{m}\right)$.

Proof. We use notation of Lemma 2, Clearly, $\bar{a}\left(\overline{u+r_{0} m_{1}}\right)=\overline{(a, m)}=\bar{\mu}_{a}$ by (44) and $\bar{a}=\bar{\mu}_{a} \bar{e}_{a}$, $\bar{e}_{a}=\left(\overline{u+r_{0} m_{1}}\right)^{-1}$. Since g.c.d. of each element from the coset $a+m R$ with the element $m$ is equal to $(a, m)$, the proof is done.

Note that the presentation of $\bar{a} \in R_{m}$ in Lemma 3 in the form $\bar{a}=\bar{\mu}_{a} \bar{e}_{a}$ is ambiguous.

Example 1. The element $\overline{4} \in \mathbb{Z}_{6}$ can be write as $\overline{4}=\overline{2} \cdot \overline{2}=\overline{2} \cdot \overline{5}$, where $2=(4,6)$ and $\overline{5} \in U\left(\mathbb{Z}_{6}\right)$, but $\overline{2} \notin U\left(\mathbb{Z}_{6}\right)$. Furthermore, $\mathbb{Z}_{36} \ni \overline{8}=\overline{4} \cdot \overline{11}=\overline{4} \cdot \overline{29}$ in which $\overline{11}, \overline{29} \in U\left(\mathbb{Z}_{36}\right)$.

Lemma 4. If $\bar{a}, \bar{b} \in R_{m}$ are multiples of each others, then $\bar{a}$ and $\bar{b}$ are associates in $R_{m}$.

Proof. If $\bar{a}=\bar{b} \bar{c}$, then there exist $a, b, c \in R$ such that $a=b c$ by Lemma 1. Set $\mu_{a}:=(a, m)$ and $\mu_{b}:=(b, m)$. Clearly, $\mu_{a}=(b c, m)$, so $\mu_{b} \mid \mu_{a}$.

Similarly, from $\bar{b}=\bar{a} \bar{d}$ follows that $\mu_{a} \mid \mu_{b}$. Since $R$ is a domain, $\mu_{a}=\mu_{b} e$, where $e \in U(R)$. Furthermore, $\bar{a}=\bar{\mu}_{a} \cdot \bar{e}_{a}$ and $\bar{b}=\bar{\mu}_{b} \cdot \bar{e}_{b}$, where $\bar{e}_{a}, \bar{e}_{b} \in U\left(R_{m}\right)$ by Lemma 3, so

$$
\bar{a}=\bar{\mu}_{a} \bar{e}_{a}=\left(\bar{\mu}_{b} \cdot \bar{e}\right) \bar{e}_{a}=\bar{\mu}_{b} \bar{e}_{b}\left(\bar{e}_{b}\right)^{-1} \bar{e} \bar{e}_{a}=\bar{b} \bar{\xi},
$$

where $\bar{\xi}=\left(\bar{e}_{b}\right)^{-1} \bar{e} \bar{e}_{a} \in U\left(R_{m}\right)$. 
Let $\bar{c} \in R_{m}$. The annihilator of $\bar{c}$ in $R_{m}$ is denoted by $\operatorname{Ann}(\bar{c})$.

Lemma 5. If $b \in R$, then $\operatorname{Ann}(\bar{b})=\bar{\alpha}_{b} R_{m}$, where $\alpha_{b}:=\frac{m}{\mu_{b}} \in R$ and $\mu_{b}:=(b, m)$.

Proof. The ideal Ann $(\bar{b})$ consists of the images of $s \in R$ for which $b s=m p$, so

$$
\frac{b}{\mu_{b}} s=\frac{m}{\mu_{b}} p \quad \text { and } \quad \frac{m}{\mu_{b}} \mid \frac{b}{\mu_{b}} s .
$$

We conclude that $\frac{m}{\mu_{b}} \mid s$ and $s=\frac{m}{\mu_{b}} s^{\prime}$ for some $s^{\prime} \in R$ because $\left(\frac{m}{\mu_{b}}, \frac{b}{\mu_{b}}\right)=1$. Consequently, $\bar{c}=\bar{\alpha}_{b} \cdot \overline{s^{\prime}} \in \bar{\alpha}_{b} R_{m}$ and $\operatorname{Ann}(\bar{b}) \subseteq \bar{\alpha}_{b} R_{m}$.

Since $b\left(\frac{m}{\mu_{b}} t\right)=m\left(\frac{b}{\mu_{b}} t\right)$ for each $t \in R$, the image of $\frac{m}{(b, m)} t$ lies in Ann $(\bar{b})$. This yields that $\bar{\alpha}_{b} R_{m} \subseteq \operatorname{Ann}(\bar{b})$, so $\operatorname{Ann}(\bar{b})=\bar{\alpha}_{b} R_{m}$.

Proof of Theorem 11. (i) Let $\bar{a}, \bar{b}, \bar{c} \in R_{m} \backslash\{0\}$ such that $\bar{a}=\bar{b} \bar{c}$ and $a=b c$ (see Lemma 1). Thus $\bar{a}=\bar{\mu}_{a} \cdot \bar{e}_{a}$ and $\bar{b}=\bar{\mu}_{b} \cdot \bar{e}_{b}$, where $\mu_{a}:=(a, m), \mu_{b}:=(b, m)$ and $\bar{e}_{a}, \bar{e}_{b} \in U\left(R_{m}\right)$ by Lemma 3. This yields

$$
\frac{\mu_{a}}{\mu_{b}}=\frac{(a, m)}{(b, m)}=\frac{(b c, m)}{(b, m)}=\left(\frac{b c}{(b, m)}, \frac{m}{(b, m)}\right)=\left(\frac{b}{(b, m)} c, \frac{m}{(b, m)}\right)=\left(c, \frac{m}{(b, m)}\right),
$$

so $\mu_{a}=\mu_{b} \sigma$, where $\sigma:=\left(c, \frac{m}{(b, m)}\right)$. It is easy to check that

$$
\bar{a}=\bar{\mu}_{a} \cdot \bar{e}_{a}=\left(\bar{\mu}_{b} \cdot \bar{e}_{b}\right)\left(\bar{\sigma} \cdot\left(\bar{e}_{b}\right)^{-1} \cdot \bar{e}_{a}\right)=\bar{b} \cdot \overline{c_{1}},
$$

where $\overline{c_{1}}=\bar{\sigma} \cdot\left(\bar{e}_{b}\right)^{-1} \cdot \bar{e}_{a}$. It follows that the set of solutions of the equation $\bar{a}=\bar{b} \cdot \bar{x}$ is

$$
\bar{c}_{1}+\operatorname{Ann}(\bar{b})=\bar{\sigma} \cdot\left(\bar{e}_{b}\right)^{-1} \cdot \bar{e}_{a}+\operatorname{Ann}(\bar{b}) .
$$

Thus Ann $(\bar{b})=\bar{\alpha}_{b} R_{m}$, where $\alpha_{b}:=\frac{m}{\mu_{b}}$ by Lemma 5, As $\sigma=\frac{\mu_{a}}{\mu_{b}}$, in which $\mu_{a} \mid m$, we get $\sigma \mid \frac{m}{\mu_{b}} R$. Therefore $\bar{\sigma} \mid \operatorname{Ann}(\bar{b})$ and $\bar{c}_{1}=\bar{\sigma} \cdot\left(\bar{e}_{b}\right)^{-1} \cdot \bar{e}_{a} \mid \operatorname{Ann}(\bar{b})$, so $\bar{c}_{1} \mid\left(\bar{c}_{1}+\operatorname{Ann}(\bar{b})\right)$. Hence $\bar{c}_{1}$ is a solution of $\bar{a}=\bar{b} \cdot \bar{x}$, and the divisor of all of the other solutions of this equation.

Note that, the solution of a solvable linear equation $\bar{a}=\bar{b} \cdot \bar{x}$ in $R_{m}$ which divides all other solutions is called generating solution of this equation.

Proof of Theorem 1(ii). Let $f, g$ be generating solutions of a linear equation $b=a x$. It follows that $f \mid g$ and $g \mid f$. From Lemma 4, we conclude that $f, g$ are associates.

Example 2. Let $R_{m}=\mathbb{Z}_{36}$. The set $\overline{6}+\operatorname{Ann}(\overline{4})=\{\overline{6}, \overline{15}, \overline{24}, \overline{33}\}$ consists of all solutions of the solvable equation $\overline{4} \bar{x}=\overline{24}$, where $\operatorname{Ann}(\overline{4})=\{\overline{0}, \overline{9}, \overline{18}, \overline{27}\}$. The elements $\overline{15}$ and $\overline{33}$ are generating solutions of our equation. These elements divide all elements from $\overline{6}+\operatorname{Ann}(\overline{4})$ and are pairwise associates, because $\overline{33}=\overline{15} \cdot \overline{31}=\overline{15} \cdot \overline{7}$, where $\overline{7}, \overline{31} \in U\left(\mathbb{Z}_{36}\right)$.

Note that the generating solutions of a linear equation can be characterized as the g.c.d. of all solutions of this equation. However, in general, the g.c.d. of two solutions is not a solution. Indeed, let $R_{m}=\mathbb{Z}_{72}$. The numbers $\overline{2}, \overline{20}, \overline{38}, \overline{56}$ are all solutions of the equation $\overline{4} \bar{x}=\overline{8}$. Obviously, $\overline{2}=(\overline{2}, \overline{20}, \overline{38}, \overline{56})$ are solutions of our equation, but $\overline{4}=(\overline{20}, \overline{56})$ is not a solution.

Recall that, if we fix an ordering relation $\leq$ on elements of the set $R_{m}$, then the set of generating solutions of each solvable equation $\bar{\varphi}_{2}=\bar{\varphi}_{1} \cdot \bar{x}$ contains a minimal element which we denote by $\frac{\bar{\varphi}_{2}}{\bar{\varphi}_{1}}$.

Corollary 1. If $\bar{\varphi}_{1}, \bar{\varphi}_{2} \in R_{m}$ such that $\bar{\varphi}_{1} \mid \bar{\varphi}_{2} \neq 0$, then $\bar{\varphi}_{2}=\frac{\bar{\varphi}_{2}}{\bar{\varphi}_{1}} \cdot \bar{\varphi}_{1}$. 
Proof. Clearly $\frac{\bar{\varphi}_{2}}{\bar{\varphi}_{1}}$ is a solution of the equation $\bar{\varphi}_{2}=\bar{\varphi}_{1} \cdot \bar{x}$, so $\bar{\varphi}_{2}=\frac{\bar{\varphi}_{2}}{\bar{\varphi}_{1}} \cdot \bar{\varphi}_{1}$.

Let $\bar{\varphi}_{1}, \bar{\varphi}_{2}, \ldots, \bar{\varphi}_{n} \in R_{m}$ such that $\bar{\varphi}_{1}\left|\bar{\varphi}_{2}\right| \cdots \mid \bar{\varphi}_{n} \neq 0$. Define the following sets:

$$
\bar{M}_{i j}:=\left\{\bar{x} \in R_{m} \mid \bar{\varphi}_{i}=\bar{\varphi}_{j} \cdot \bar{x}\right\}, \quad(1 \leq j<i \leq n) .
$$

Lemma 6. For all $\bar{\mu}_{i j} \in \bar{M}_{i j}(1 \leq j<i \leq n)$ (see (5) ) the following holds:

$$
\bar{x}_{p, k}:=\bar{\mu}_{p, p-1} \cdot \bar{\mu}_{p+1, p} \cdots \bar{\mu}_{p+k, p+k-1} \in \bar{M}_{p+k, p-1}, \quad(1<p \leq n, \quad 0 \leq k \leq n-p) .
$$

Proof. Indeed, using the definition of $\bar{M}_{i j}$, we have

$$
\begin{aligned}
\bar{\varphi}_{p-1} \cdot \bar{x}_{p, k} & =\left(\bar{\varphi}_{p-1} \cdot \bar{\mu}_{p, p-1}\right) \bar{\mu}_{p+1, p} \cdots \bar{\mu}_{p+k, p+k-1} \\
& =\left(\bar{\varphi}_{p} \cdot \bar{\mu}_{p+1, p}\right) \bar{\mu}_{p+2, p+1} \cdots \bar{\mu}_{p+k, p+k-1} \\
& =\cdots= \\
& =\bar{\varphi}_{p+k-1} \cdot \bar{\mu}_{p+k, p+k-1} \\
& =\bar{\varphi}_{p+k} .
\end{aligned}
$$

Consequently, $\bar{x}_{p, k} \in \bar{M}_{p+k, p-1}$.

Lemma 7. Let $\bar{\varphi}_{1}, \bar{\varphi}_{2}, \bar{\varphi}_{3} \in R_{m}$. If $\bar{\varphi}_{1}\left|\bar{\varphi}_{2}\right| \bar{\varphi}_{3} \neq 0$, then

$$
\frac{\bar{\varphi}_{2}}{\bar{\varphi}_{1}} \cdot \frac{\bar{\varphi}_{3}}{\bar{\varphi}_{2}}=\frac{\bar{\varphi}_{3}}{\bar{\varphi}_{1}} \cdot \bar{e} \in \bar{M}_{31}, \quad\left(\bar{e} \in U\left(R_{m}\right)\right) .
$$

Moreover, $\frac{\bar{\varphi}_{3}}{\bar{\varphi}_{1}} \cdot \bar{e}$ is a generating solution of $\bar{\varphi}_{3}=\bar{\varphi}_{1} \bar{x}$.

Proof. There exist $\varphi_{1}, \varphi_{2}, \varphi_{3} \in R$ which are preimages of $\bar{\varphi}_{1}, \bar{\varphi}_{2}, \bar{\varphi}_{3}$, such that $\varphi_{1}\left|\varphi_{2}\right| \varphi_{3} \neq 0$ by Lemma 1. Clearly $\bar{\varphi}_{i}=\bar{\mu}_{\varphi_{i}} \bar{e}_{\varphi_{i}}$, in which $\bar{e}_{\varphi_{i}} \in U\left(R_{m}\right)$ and $\mu_{\varphi_{i}}:=\left(\varphi_{i}, m\right)$ by Lemma 3, Using the same argument, as in the proof of Theorem 1(i), we get that each generating solution of the linear equation $\bar{\varphi}_{i}=\bar{\varphi}_{j} \bar{x}$ has the following form

$$
\bar{\psi}_{i j}:=\overline{\left(\frac{\mu_{\varphi_{i}}}{\mu_{\varphi_{j}}}\right)} \cdot \bar{e}_{i} \cdot\left(\bar{e}_{j}\right)^{-1},
$$

in which $\bar{e}_{i}, \bar{e}_{j} \in U\left(R_{m}\right)$ and $1 \leq j<i \leq n$. Since $\varphi_{1}\left|\varphi_{2}\right| \varphi_{3} \neq 0$,

$$
\frac{\mu_{\varphi_{2}}}{\mu_{\varphi_{1}}} \cdot \frac{\mu_{\varphi_{3}}}{\mu_{\varphi_{2}}}=\frac{\mu_{\varphi_{3}}}{\mu_{\varphi_{1}}} \quad \text { and } \overline{\left(\frac{\mu_{\varphi_{2}}}{\mu_{\varphi_{1}}}\right)} \cdot \overline{\left(\frac{\mu_{\varphi_{3}}}{\mu_{\varphi_{2}}}\right)}=\overline{\left(\frac{\mu_{\varphi_{3}}}{\mu_{\varphi_{1}}}\right)} \text {. }
$$

This yields that

$$
\bar{\psi}_{21} \bar{\psi}_{32}=\bar{\psi}_{31}
$$

According to Theorem 1 (ii),

$$
\frac{\bar{\varphi}_{2}}{\frac{\bar{\varphi}_{1}}{2}}=\bar{\psi}_{21} \cdot \bar{\varepsilon}_{21}, \quad \frac{\bar{\varphi}_{3}}{\bar{\varphi}_{2}}=\bar{\psi}_{32} \cdot \bar{\varepsilon}_{32}, \quad \frac{\bar{\varphi}_{3}}{\bar{\varphi}_{1}}=\bar{\psi}_{31} \cdot \bar{\varepsilon}_{31}, \quad\left(\bar{\varepsilon}_{i j} \in U\left(R_{m}\right)\right)
$$

and $\frac{\bar{\varphi}_{2}}{\bar{\varphi}_{1}} \cdot \frac{\bar{\varphi}_{3}}{\bar{\varphi}_{2}}=\frac{\bar{\varphi}_{3}}{\bar{\varphi}_{1}} \cdot \bar{e}$ where $\bar{e}:=\left(\bar{\varepsilon}_{31}\right)^{-1} \cdot \bar{\varepsilon}_{21} \cdot \bar{\varepsilon}_{32} \in U\left(R_{m}\right)$. Since $\frac{\bar{\varphi}_{2}}{\bar{\varphi}_{1}} \cdot \frac{\bar{\varphi}_{3}}{\bar{\varphi}_{2}} \in \bar{M}_{31}$ by Lemma 6. $\frac{\bar{\varphi}_{3}}{\bar{\varphi}_{1}} \cdot \bar{e} \in \bar{M}_{31}$. The element $\frac{\bar{\varphi}_{3}}{\bar{\varphi}_{1}}$ is a generating solution of $\bar{\varphi}_{3}=\bar{\varphi}_{1} \bar{x}$. According to Theorem 1(ii), the element $\frac{\bar{\varphi}_{3}}{\bar{\varphi}_{1}} \cdot \bar{e}$ is also a generating solution of the same equation.

Proving the previous lemma, we obtained (6), which can be formulated as the following independent result. 
Corollary 2. Each set $\bar{M}_{i j}$ (see (5) ) contains a generating solution $\bar{\psi}_{i j}$ such that

$$
\bar{\psi}_{i, i-1} \cdot \bar{\psi}_{i+1, i}=\bar{\psi}_{i+1, i-1}, \quad(2 \leq i<n-1) .
$$

Example 3. Note that, a product of solutions of two equations $\bar{\varphi}_{2}=\bar{\varphi}_{1} \bar{x}$ and $\bar{\varphi}_{3}=\bar{\varphi}_{2} \bar{x}$, in which at least one factor is a non generated solution, in general is not a generating solution of $\bar{\varphi}_{3}=\bar{\varphi}_{1} \bar{x}$.

Indeed, let $R_{m}=\mathbb{Z}_{72}$ and let $\left\{\bar{\varphi}_{1}, \bar{\varphi}_{2}, \bar{\varphi}_{3}\right\}=\{\overline{4}, \overline{8}, \overline{24}\}$.

\begin{tabular}{|c|c|c|}
\hline Equation & Solutions & Generating solutions \\
\hline$\overline{4} \bar{x}=\overline{8}$ & $\overline{2}, \overline{20}, \overline{38}, \overline{56}$ & $\overline{\mathbf{2}}, \overline{38}$ \\
\hline$\overline{8} \bar{x}=\overline{24}$ & $\overline{3}, \overline{\mathbf{1 2}}, \overline{21}, \overline{30}, \overline{39}, \overline{48}, \overline{57}, \overline{66}$ & $\overline{3}, \overline{21}, \overline{39}, \overline{57}$ \\
\hline$\overline{4} \bar{x}=\overline{24}$ & $\overline{6}, \overline{24}, \overline{42}, \overline{60}$ & $\overline{6}, \overline{42}$ \\
\hline
\end{tabular}

However, $\overline{2} \cdot \overline{12}=\overline{24}$ is not a generating solution of the equation $\overline{24}=\overline{4} \cdot \bar{x}$.

Lemma 8. Let $\bar{a}, \bar{b} \in R_{m}$. If $\bar{b} \mid \bar{a}$ then $\quad \frac{\bar{a}}{\bar{b}}=\frac{\bar{\alpha}_{b}}{\bar{\alpha}_{a}} \cdot \bar{e}$ in which

$$
\bar{\alpha}_{a}:=\overline{\left(\frac{m}{(a, m)}\right)}, \quad \bar{\alpha}_{b}: \overline{\left(\frac{m}{(b, m)}\right)}, \quad \text { and } \quad \bar{e} \in U\left(R_{m}\right) .
$$

Proof. Let $\mu_{a}:=(a, m)$ and $\mu_{b}:=(b, m)$. This yields that

$$
\alpha_{b}=\frac{m}{\mu_{b}}=\frac{m}{\mu_{a}} \frac{\mu_{a}}{\mu_{b}}=\alpha_{a} \frac{\mu_{a}}{\mu_{b}}
$$

and $\bar{\alpha}_{b}=\bar{\alpha}_{a} \cdot \bar{\sigma}$ in which $\sigma:=\frac{\mu_{a}}{\mu_{b}}$. Therefore, $\bar{\sigma}+\operatorname{Ann}\left(\bar{\alpha}_{a}\right)$ is the set of solutions of the equation $\bar{\alpha}_{b}=\bar{\alpha}_{a} \bar{x}$. The ideal $\operatorname{Ann}\left(\bar{\alpha}_{a}\right)$ is generated by the image of $\alpha_{a}:=\frac{m}{\mu_{a}} \in R$ (see Lemma 5 ), so

$$
\frac{m}{\left(\frac{m}{\mu_{a}}, m\right)}=\frac{m}{\frac{m}{\mu_{a}}}=\mu_{a} \quad \text { and } \quad \operatorname{Ann}\left(\bar{\alpha}_{a}\right)=\bar{\mu}_{a} R_{m} .
$$

Clearly $\bar{\mu}_{a}=\bar{\mu}_{b} \bar{\sigma}$ and $\bar{\sigma} \mid \bar{\mu}_{a}$. This yields that $\bar{\sigma}$ is a generating solution of the class $\bar{\sigma}+\operatorname{Ann}\left(\bar{\alpha}_{a}\right)$. Consequently, $\quad \frac{\bar{a}}{\bar{b}}=\frac{\bar{\alpha}_{b}}{\bar{\alpha}_{a}} \cdot \bar{e}$ for some $\bar{e} \in U\left(R_{m}\right)$.

In order to simplify the notation, in the sequel of the paper we will omit the over line when referring to the elements of the ring $R_{m}$.

To a permutation $\sigma=\left(\begin{array}{cccc}1 & 2 & \ldots & n \\ i_{1} & i_{2} & \ldots & i_{n}\end{array}\right) \in S_{n}$ we assign the following two sets:

$$
\begin{aligned}
& \mathfrak{I}_{1}(\sigma)=\left\{\left(p_{i}, q_{i}\right) \mid p_{i}>q_{i} \text { and }\left(\begin{array}{c}
p_{i} \\
q_{i}
\end{array}\right) \text { is a column in } \sigma\right\} ; \\
& \mathfrak{I}_{2}(\sigma)=\left\{\left(\alpha_{i}, \beta_{i}\right) \mid \alpha_{i} \leq \beta_{i} \text { and }\left(\begin{array}{c}
\alpha_{i} \\
\beta_{i}
\end{array}\right) \text { is a column in } \sigma\right\} .
\end{aligned}
$$

Lemma 9. If $\sigma \in S_{n}$, then

$$
\prod_{\left(p_{i}, q_{i}\right) \in \mathfrak{I}_{1}(\sigma)} \frac{p_{i}}{q_{i}}=\prod_{\left(\alpha_{i}, \beta_{i}\right) \in \mathfrak{I}_{2}(\sigma)} \frac{\beta_{i}}{\alpha_{i}} .
$$

Moreover, if $\Phi=\operatorname{diag}\left(\varphi_{1}, \ldots, \varphi_{n}\right) \in R_{m}^{n \times n}$ such that $\varphi_{1}\left|\varphi_{2}\right| \cdots \mid \varphi_{n} \neq 0$, then each set $\bar{M}_{i j}$ (see (5)) contains a generating solution $\psi_{i j}$ (with $1 \leq j<i \leq n$ ), such that

$$
\prod_{\left(p_{i}, q_{i}\right) \in \mathfrak{I}_{1}(\sigma)} \psi_{p_{i}, q_{i}}=\prod_{\left(\alpha_{i}, \beta_{i}\right) \in \mathfrak{I}_{2}(\sigma)} \psi_{\beta_{i}, \alpha_{i}} .
$$


Proof. Let us prove (7). Let $\sigma_{t}=\left(i_{1}, i_{2}, \ldots, i_{t}\right)$ be a cycle. The case $t=2$ is trivial. Now let (7) holds for $\sigma_{t}$ where $t>2$. Consider the cycle $\sigma_{t+1}=\left(i_{1}, i_{2}, \ldots, i_{t}, i_{t+1}\right)$ of length $t+1$ which we obtain from $\sigma_{t}$ adding a new symbol $i_{t+1}$. Let us prove that the equation (7) holds for the new cycle $\sigma_{t+1}$, if we replace the pair $\left(i_{t}, i_{1}\right)$ by two pairs $\left(i_{t}, i_{t+1}\right)$ and $\left(i_{t+1}, i_{1}\right)$ adding them in appropriate places of the equation (17). These three pairs are either lie in one set $\mathfrak{I}_{s_{1}}$ or two are in $\mathfrak{I}_{s_{2}}$ and the one is in $\mathfrak{I}_{s_{3}}$, where $s_{1}, s_{2}, s_{3} \in\{1,2\}$ and $s_{2} \neq s_{3}$. Now considering these possible cases, it is easy to check that (7) holds for any cycle of finite length. Since each permutation is a product of disjoint cycles, (7) always holds.

The proof of (8) follows immediately from (7), Lemma 6 and Corollary 2 ,

Lemma 10. If $\psi_{i j}$ is such a generating solution of $\bar{M}_{i j}$ for all $1 \leq j<i \leq n$ for which (8) holds, then

$$
\operatorname{det}\left[\begin{array}{ccccc}
h_{11} & h_{12} & \cdots & h_{1, n-1} & h_{1 n} \\
\psi_{21} h_{21} & h_{22} & \cdots & h_{2, n-1} & h_{2 n} \\
\cdots & \cdots & \cdots & \cdots \\
\psi_{n 1} h_{n 1} & \psi_{n 2} h_{n 2} & \cdots & \psi_{n, n-1} h_{n, n-1} & h_{n n}
\end{array}\right]=\operatorname{det}\left[\begin{array}{ccccc}
h_{11} & \psi_{21} h_{12} & \cdots & \psi_{n-1,1} h_{1, n-1} & \psi_{n 1} h_{1 n} \\
h_{21} & h_{22} & \cdots & \psi_{n-1,2} h_{2, n-1} & \psi_{n 2} h_{2 n} \\
\cdots & \ldots & \cdots & \cdots & \cdots \\
h_{n 1} & h_{n 2} & \cdots & h_{n, n-1} & h_{n n}
\end{array}\right],
$$

in which $h_{i j} \in R_{m}$.

Proof. Let us show that both determinants consist of the same summands. Assign to each summand a permutation $\sigma=\left(\begin{array}{cccc}1 & 2 & \ldots & n \\ i_{1} & i_{2} & \ldots & i_{n}\end{array}\right)$. In the left hand side determinant these summands have the following form:

$$
(-1)^{\operatorname{sign}(\sigma)} \psi_{p_{1}, q_{1}} \psi_{p_{2}, q_{2}} \ldots \psi_{p_{s}, q_{s}} h_{p_{1}, q_{1}} \ldots h_{p_{s}, q_{s}} h_{\alpha_{1}, \beta_{1}} \ldots h_{\alpha_{t}, \beta_{t}},
$$

where $\left(p_{1}, q_{1}\right), \ldots,\left(p_{s}, q_{s}\right) \in \mathfrak{I}_{1}(\sigma),\left(\alpha_{1}, \beta_{1}\right), \ldots,\left(\alpha_{t}, \beta_{t}\right) \in \mathfrak{I}_{2}(\sigma), s=\left|\mathfrak{I}_{1}(\sigma)\right|$ and $t=\left|\mathfrak{I}_{2}(\sigma)\right|$. The corresponding summands in the right hand side determinant are

$$
(-1)^{\operatorname{sign}(\sigma)} \psi_{\beta_{1}, \alpha_{1}} \psi_{\beta_{2}, \alpha_{2}} \ldots \psi_{\beta_{t}, \alpha_{t}} h_{p_{1}, q_{1}} \ldots h_{p_{s}, q_{s}} h_{\alpha_{1}, \beta_{1}} \ldots h_{\alpha_{t}, \beta_{t}} .
$$

Finally, these terms are the same by (8) from Lemma 9 ,

Lemma 11. Let $\Phi=\operatorname{diag}\left(\varphi_{1}, \ldots, \varphi_{n}\right) \in R_{m}^{n \times n}$ such that $\varphi_{1}\left|\varphi_{2}\right| \cdots \mid \varphi_{n} \neq 0$. If $\alpha_{i j}$ and $\beta_{i j}$ are arbitrary elements from $\bar{M}_{i j}$ for all $1 \leq j<i \leq n$, then

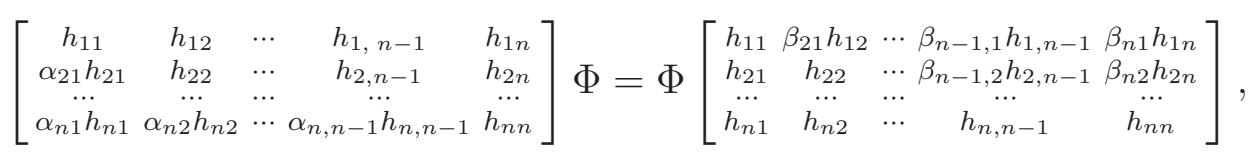

in which $h_{i j} \in R_{m}$.

Proof. Each element at position $(i, j)$ where $i>j$ of the matrix from the left hand side product has the form $\varphi_{j} \alpha_{i j} h_{i j}$. The corresponding element of the matrix from the right hand side product has the form $\varphi_{i} h_{i j}$. Since $\alpha_{i j}$ is a solution of the equation $\varphi_{i}=\varphi_{j} x$, we can replace $\varphi_{i}$ by $\varphi_{j} \alpha_{i j}$. This means that the elements of the matrices which are situated below the main diagonals coincide.

Let $i<j$. Each element at position $(i j)$ of the matrix from the left hand side product has the form $\varphi_{j} h_{i j}$. The corresponding element of the matrix from the right hand side product has the form $\varphi_{i} \beta_{j i} h_{i j}$, respectively. As above, $\varphi_{j}=\varphi_{i} \beta_{j i}$. Taking into account that main diagonals of both matrices are equal, we obtain the requested equality.

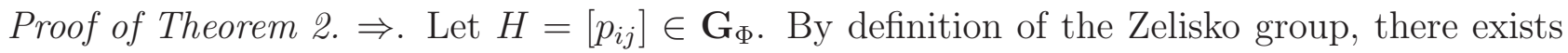
$S=\left[s_{i j}\right] \in \mathrm{GL}_{n}\left(R_{m}\right)$ such that $H \Phi=\Phi S$, so

$$
\varphi_{j} p_{i j}=\varphi_{i} s_{i j}, \quad(1 \leq i, j \leq n) .
$$


Obviously, for $i \leq j$ no restrictions are imposed on the elements $p_{i j}$.

Let $i>j$. Since $\varphi_{j} \mid \varphi_{i}$, we have $\varphi_{i}=\varphi_{j} \cdot \frac{\varphi_{i}}{\varphi_{j}}$ by Corollary 1. Now using (9) we obtain that

$$
\varphi_{j}\left(p_{i j}-\frac{\varphi_{i}}{\varphi_{j}} s_{i j}\right)=0
$$

So $q_{i j}:=p_{i j}-\frac{\varphi_{i}}{\varphi_{j}} s_{i j} \in \operatorname{Ann}\left(\varphi_{j}\right)$. This yields that $p_{i j} \in \frac{\varphi_{i}}{\varphi_{j}} s_{i j}+\operatorname{Ann}\left(\varphi_{j}\right)$ and $\operatorname{Ann}\left(\varphi_{j}\right)=\alpha_{\varphi_{j}} R_{m}$ by Lemma 5. Furthermore, $\frac{\varphi_{i}}{\varphi_{j}}=\frac{\alpha_{\varphi_{j}}}{\alpha_{\varphi_{i}}} \cdot e$ (see Lemma 8), in which $e \in U\left(R_{m}\right)$. It follows that

$$
\alpha_{\varphi_{j}}=\frac{\alpha_{\varphi_{j}}}{\alpha_{\varphi_{i}}} \cdot \alpha_{\varphi_{i}}=\left(\frac{\varphi_{i}}{\varphi_{j}} \cdot e^{-1}\right) \alpha_{\varphi_{i}}
$$

by Corollary 1, so $\frac{\varphi_{i}}{\varphi_{j}} \mid \alpha_{\varphi_{j}}$. Thus, $\frac{\varphi_{i}}{\varphi_{j}}$ is a divisor of all elements of the ideal $\alpha_{\varphi_{j}} R_{m}=\operatorname{Ann}\left(\varphi_{j}\right)$. This yields that $\frac{\varphi_{i}}{\varphi_{j}} \mid q_{i j}$ and $q_{i j}=\frac{\varphi_{i}}{\varphi_{j}} \cdot l_{i j}$ for some $l_{i j} \in R_{m}$. Hence,

$$
p_{i j}=\frac{\varphi_{i}}{\varphi_{j}} s_{i j}+q_{i j}=\frac{\varphi_{i}}{\varphi_{j}} s_{i j}+\frac{\varphi_{i}}{\varphi_{j}} l_{i j}=\frac{\varphi_{i}}{\varphi_{j}}\left(s_{i j}+l_{i j}\right)=\frac{\varphi_{i}}{\varphi_{j}} h_{i j},
$$

in which $h_{i j}:=s_{i j}+l_{i j}$. Therefore, the matrix $H=\left[p_{i j}\right]$ has the form (2)).

$\Leftarrow$. The sequential product of generating solutions of the sets $\bar{M}_{i, i-1}, \bar{M}_{i-1, i-2}, \ldots, \bar{M}_{j+1, j}$ (see (5)) is denoted by

$$
\psi_{i j}:=\frac{\varphi_{i}}{\varphi_{i-1}} \cdot \frac{\varphi_{i-1}}{\varphi_{i-2}} \cdots \frac{\varphi_{j+2}}{\varphi_{j+1}} \cdot \frac{\varphi_{j+1}}{\varphi_{j}}, \quad(1 \leq j<i \leq n) .
$$

Each $\psi_{i j}$ is the generating solution of $\bar{M}_{i j}$ by Lemma 7. Moreover, $\frac{\varphi_{i}}{\varphi_{j}}=\psi_{i j} e_{i j}$ in which $e_{i j} \in U\left(R_{m}\right)$. Hence, we have a presentation

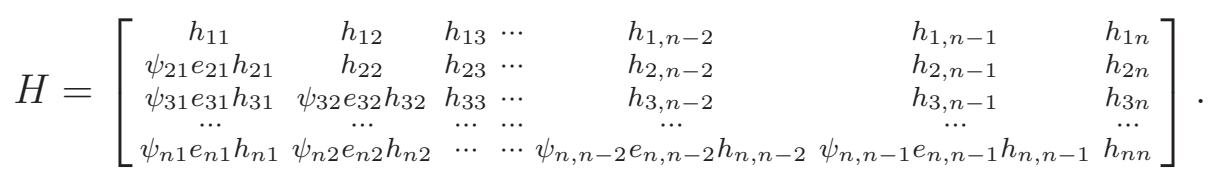

According to Lemma 10, the determinants of the matrix $H$ and the matrix

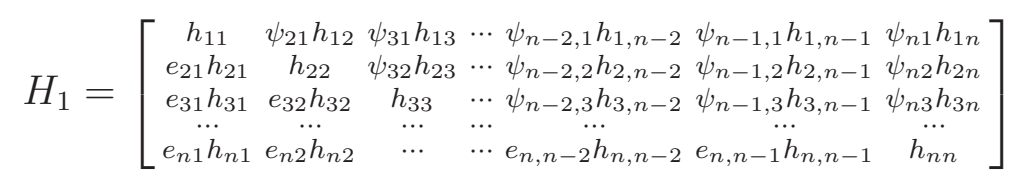

coincide, so $H_{1}$ is invertible. Now, using Lemma 11 gives $H \Phi=\Phi H_{1}$, so $H \in \mathbf{G}_{\Phi}$.

We propose the following.

Problem. Describe that rings $R$ in which g.c.d. of all solutions of a solvable linear equation $b=a x(a, b \in R)$ in $R$ is again a solution of the same linear equation.

Note that, for rings $M_{n}(R)$ over elementary divisor domains $R$ a positive solution to this problem was done in [14].

\section{ACKnowledgement}

Authors would like to express their gratitude to the referee for valuable remarks. The work was supported by the UAEU UPAR [grant number G00002160]. 


\section{REFERENCES}

[1] D. D. Anderson and J. R. Juett. Stable range and almost stable range. J. Pure Appl. Algebra, 216(10):20942097, 2012.

[2] H. Bass. K-theory and stable algebra. Inst. Hautes Études Sci. Publ. Math., (22):5-60, 1964.

[3] V. A. Bovdi and V. P. Shchedryk. Commutative Bezout domains of stable range 1.5. Linear Algebra Appl., 568:127-134, 2019.

[4] H. Chen. On simple factorization of invertible matrices. Linear Multilinear Algebra, 55(1):81-92, 2007.

[5] H. Chen. Rings related to stable range conditions, volume 11 of Series in Algebra. World Scientific Publishing Co. Pte. Ltd., Hackensack, NJ, 2011.

[6] I. Kaplansky. Elementary divisors and modules. Trans. Amer. Math. Soc., 66:464-491, 1949.

[7] P. S. Kazīmīrskiı̌. A solution to the problem of separating a regular factor from a matrix polynomial. Ukrain. Mat. Ž., 32:483-498, 1980.

[8] W. W. McGovern. Bézout rings with almost stable range 1. J. Pure Appl. Algebra, 212(2):340-348, 2008.

[9] K. R. Nagarajan, M. P. Devasahayam, and T. Soundararajan. Products of three triangular matrices over commutative rings. Linear Algebra Appl., 348:1-6, 2002.

[10] V. Shchedryk. Factorization of matrices over elementary divisor rings. Graduate Texts in Mathematics, http://www.iapmm.lviv.ua/14/index_e.htm. Lviv, Pidstryhach Institute for Applied Problems of Mechanics and Mathematics of the NAS of Ukraine, 2017.

[11] V. P. Shchedryk. Some properties of primitive matrices over Bezout B-domain. Algebra Discrete Math., (2):46-57, 2005.

[12] V. P. Shchedryk. Bezout rings of stable range 1.5. Ukrainian Math. J., 67(6):960-974, 2015. Translation of Ukraïn. Mat. Zh. 67 (2015), no. 6, 849-860.

[13] V. P. Shchedryk. Bezout rings of stable rank 1.5 and the decomposition of a complete linear group into products of its subgroups. Ukrä̈n. Mat. Zh., 69(1):113-120, 2017.

[14] V. P. Shchedryk. A greatest common divisor and a least common multiple of solutions of a linear matrix equation. Ukraïn. Mat. Zh., arXiv:2011.05173(to accepted):1-12, 2021.

[15] L. N. Vaserstein and E. Wheland. Commutators and companion matrices over rings of stable rank 1. Linear Algebra Appl., 142:263-277, 1990.

[16] V. R. Zelīsko. Construction of a class of invertible matrices. Mat. Metody i Fiz.-Mekh. Polya, 12:14-21, 120,1980 .

United Arab Emirates University, Al Ain, UAE

Email address: vbovdi@gmail.com

Pidstryhach Institute for Applied Problems of Mechanics and Mathematics, National AcadEMY OF SCIEnCES OF Ukraine, LVIV, UkRAine

Email address: shchedrykv@ukr.net 